\title{
Artículos
}

\section{Edición digital: formatos y alternativas}

\author{
Por Pedro Hípola y Ricardo Eíto Brun
}

\begin{abstract}
Resumen: La gran cantidad de formatos existentes en la actualidad para

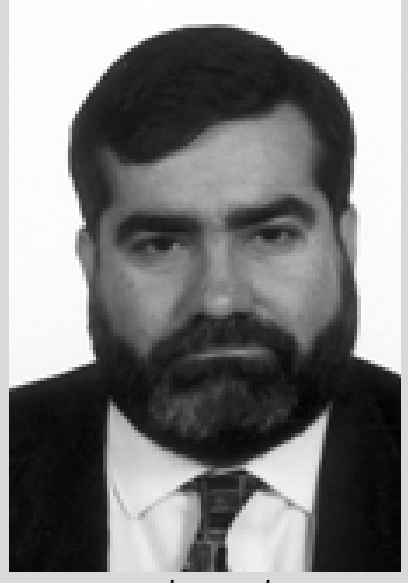

Pedro Hípola desarrollar documentos digitales lleva, en ocasiones, a analizar y evaluar profundamente las prestaciones que cada uno presenta. Su accesibilidad, portabilidad y métodos para su creación se convierten en puntos esenciales a tener en cuenta a la hora de elegir entre uno u otro. Esta situación trae consigo la búsqueda de estándares lo más ampliamente aceptados posible que permitan su máximo desarrollo en el futuro, y que los documentos que han sido ya creados no se queden incompatibles con los nuevos formatos. En este artículo se hace un repaso esencial a todos aquellos que en los últimos años han ido apareciendo, prestando especial atención a xml como uno de los formatos más interesantes desarrollados para internet y como posible sucesor de html.

Palabras clave: Edición digital, Xml, Sgml, Formatos de documento digital, Accesibilidad al documento digital.

Title: Digital publishing: formats and alternatives

Abstract: The large variety of formats currently used for creating digital

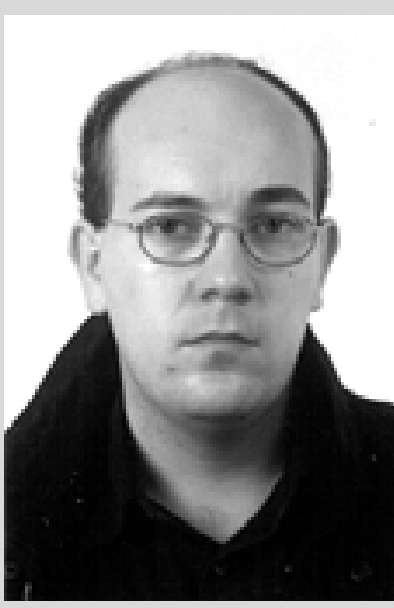

Ricardo Eíto Brun documents calls for a detailed analysis and evaluation of their functionality. Accessibility, portability and methods of creation are essential factors to consider when selecting a format. Choosing the appropriate format involves a search for a widely accepted standard to ensure that the documents won't become incompatible with new formats developed in the future. The present article reviews all of the formats which have appeared in recent years, with special attention given to XML, one of the more promising, created for internet and a likely successor to HTML.
\end{abstract}

Keywords: Digital publishing, Xml, Sgml, Digital document formats, Digital document access.

Hípola, Pedro; Eíto Brun, Ricardo. “Edición digital: formatos y alternativas”. En: El profesional de la información, 2000, octubre, v. 9, n. 10, pp 4-14.

Involucrarse de lleno en el mercado de la edición digital implica tomar decisiones sobre el formato de documento digital que se va a emplear. Durante mucho tiempo ha sido normal escuchar a los directivos de las empresas editoriales tradicionales preguntas como: ¿qué aplicaciones y formatos debemos utilizar?, ¿qué alternativas soportan con más facilidad y economía el ciclo de vida de los documentos?

Hasta mediados de los noventa la diversidad de los formatos existentes fue una auténtica fuente de obstáculos para que se pudiera llevar a cabo un intercambio fluido y económico de la información documental en soporte digital. La elección de uno u otro era crítica, ya que los productos de información digital podían llegar a quedar aislados del resto por no ajustarse a unas normas que permitiesen su gestión homogénea y aseguraran un mínimo de portabilidad. El acceso universal que iba a facilitar la difusión digital de contenidos podía verse comprometido y la inexistencia de estándares suficientemente aceptados podía conducir a la si- guiente paradoja: las dificultades que tendrían los usuarios para acceder a la información serían directamente proporcionales al volumen de documentos disponibles en formato digital.

Hoy día la situación se ha clarificado bastante. Podríamos resumir la oferta de formatos de documento digital abierto (no propietario) y avanzado (más allá de las prestaciones elementales de normas sencillas tipo ascii) en los dos principales: primero parecía que iba a reinar PDF, luego triunfó html (y, por supuesto, dentro de los formatos propietarios hay casi una hegemonía del formato Microsoft Word). Con el predominio del estándar html es indudable que se ha abierto un nuevo estilo de trabajo en la tarea de conseguir la normalización del documento digital. De todas formas, al poco tiempo de generalizarse se hicieron manifiestas sus limitaciones. $\mathrm{Pa}$ ra solucionarlas los fabricantes de navegadores desarrollaron extensiones propietarias del lenguaje incompatibles entre sí, con lo que volvió a surgir una nueva variante del problema del intercambio de información. 
Entre las litimas soluciones propuestas, el lenguaje xml (extensible markup language) se presenta como una gran promesa que gradualmente va ganando adeptos, si bien es verdad que ha sido cuestionado en algunos sectores. Sea cual sea la situación actual, parece claro que queda siempre en pie un presupuesto fundamental: la necesidad de disponer de estándares en el campo de la edición digital lo más ampliamente aceptados posible. Dado que estamos en un proceso que evidentemente no ha llegado a su fin, creemos que puede ser interesante identificar las principales direcciones por las que se ha avanzado en estos lítimos añs, lo cual puede servir no sólo para resaltar los aciertos y errores cometidos sino también para intentar predecir, en la medida de lo posible, cuáles son las líneas en las que queda más trabajo pendiente de llevar a cabo.

\section{«Frente a las alternativas basa- das en marcas descriptivas, los documentos sgml pueden ser procesados fácilmente por la aplicación que los recibe, lee o interpreta»}

En los siguientes apartados se describen algunas de las distintas alternativas disponibles para la edición digital en el camino hacia la normalización. Para ello hemos partido de un esquema que agrupa los avances ya conseguidos en nueve tendencias principales (v. Information world en español, n. 31, pp. 1-8):

Ła opción semántica

\section{Sistemas $D I P$}

Đocumentos compuestos estáticos

Đocumentos compuestos dinámicos

El paradigma hipertexto

Tecnologías para la interacción.

Conviene señlar que nuestro objetivo no es clasificar los diferentes tipos de formatos existentes, sino más bien destacar los hitos que se pueden observar en una evolución histórica caracterizada por idas y venidas, por muchos vaivenes. $\mathrm{Y}$ es evidente que no estamos hablando de grupos o tendencias excluyentes pues, por ejemplo, un mismo formato de documento digital normalmente explota características de más de una de estas tendencias. Además, a lo largo de su ciclo de vida un único documento (entendiendo como tal un mismo contenido informativo) puede representarse en múltiples formatos para satisfacer distintas necesidades.
En estas páginas también resaltaremos la utilidad de otra tecnología, la de los visores universales, que ofrece una vía más para abordar la integración de formatos heterogéneos.

\section{La opción semántica}

Con este término nos referimos a una serie de formatos que introducen etiquetas en el contenido del documento no sólo con el propósito de diferenciar sus partes significativas y hacer explícita su estructura sino también para asignar un valor semántico a cada una de sus partes, Su inclusión en el documento no es en sí el factor diferenciador de estos formatos. De hecho, todos los formatos digitales mínimamente avanzados intercalan marcas en los documentos. La principal diferencia está en el propósito con el que se añden. Por ejemplo, en los utilizados por los procesadores de textos convencionales la mayor parte de ellas indican cómo se debe formatear el documento cuando se muestra en pantalla o se imprime. Es decir, casi nunca son representativas de su estructura ni suelen asignar un valor funcional a las diferentes partes y por supuesto son propietarias, definidas por el fabricante de cada sistema de edición.

El uso de marcas para asignar valores semánticos a las partes que componen un fichero informático no es precisamente una novedad reciente. Las etiquetas que identifican los campos de una base de datos son un ejemplo de este método de trabajo que cuenta ya con décadas de explotación.

Dentro de los sistemas que se basan en el etiquetado de los campos es necesario hacer una mención especial a la estructura de un formato clásico en el entorno bibliotecario: Marc (machine readable cataloguing), esquema de registro empleado fundamentalmente para codificación e intercambio de registros catalográficos y que está regulado por la norma $I S O$ 2709. Como es sabido, su origen se remonta al a ã 1965, cuando la Library of Congress aprobó un formato interno para la codificación de registros catalográficos legibles por ordenador: Lcmarc. El lítimo paso en su evolución es el llamado Marc21, anunciado recientemente y conocido como el Marc del nuevo siglo. Entre las principales aportaciones de esta versión está la unificación de las versiones canadiense y norteamericana (Canmarc y Usmarc) así como la inclusión de una etiqueta que permite el acceso a los documentos digitales: el campo 856.

Pero, como se sabe, Marc no es propiamente un sistema de edición digital. Si lo hemos citado aquí es porque constituye un ejemplo internacional de uso de estándares para la estructuración e identificación funcional de las partes de un documento digital. 


\section{Sgml: el rey de la edición digital}

Este lenguaje es, sin lugar a dudas, la iniciativa más emblemática en el camino hacia la normalización del documento digital y también es el formato que más explota lo que hemos denominado la opción semántica. Establece:

ta-sintaxis que se debe utilizar para diseãr un conjunto de marcas aplicables a cada tipo de documento, llamado DTD (document type definition), y

taforma en la que se deben intercalar marcas en el texto de un documento para identificar las distintas partes de su estructura.

\section{«La generalización de la Red y de las intranets ha obligado a los fabricantes de los formatos de réplica a estudiar las posibi- lidades de integrarse con el len- guaje html»}

Cada tipo de documento tiene sus propias peculiaridades y necesidades de tratamiento. Sgml ofrece respuesta a este problema con la posibilidad de diseãr $D T D$ s específicas para cada caso. Una $D T D$ establece la estructura de un tipo de documento, de qué elementos va a constar, en qué orden deben situarse, qué valores deben recoger, posibilidad de ser repetidos y qué elementos pueden contener a otros. Por ejemplo, todos los documentos de tipo informe se codificarían de acuerdo a una misma $D T D$, las citas bibliográficas segú otra, etc.

En sgml se utiliza el término áplicación”para denominar a las DTDs creadas de acuerdo con las especificaciones de la norma. Los documentos que se escriben utilizando una DTD específica se llaman instancias de la DTD. Para que un sistema informático sea capaz de procesar un documento sgml es necesario distribuir, junto a su texto, la DTD que está utilizando.

Entre las ventajas que ofrece sgml hay que destacar su carácter no propietario, la independencia de aplicaciones software y de plataformas hardware específicas, la posibilidad de aplicar un control estricto sobre el contenido de los textos y la independencia entre el contenido textual del documento, su estructura y su presentación.

El origen del sistema se encuentra en el lenguaje de marcas GML, desarrollado por IBM en 1969 bajo la dirección de Charles Goldfarb. Junto a él participaron en el desarrollo Edward Mosher y Raymond Lorie (de las iniciales de sus apellidos procede el nombre del lenguaje). Las ideas detrás de la norma se encuentran en trabajos previos de William Tunnicliffe, presidente del GCA Composition Committee, y Stanley Rice.

La primera aplicación desarrollada para utilizar dicho sistema fue la IBM document composition facility, conocida como script, que incluía un conjunto de etiquetas genéricas predefinidas. Segú Truly Donovan, este grupo de etiquetas iniciales hicieron que se confundiese la filosofía abierta del proyecto con un conjunto limitado de marcas.

Sgml evolucionó hasta convertirse en el estándar internacional ISO 8879:1986 "Information processing-Text and office systems-Standard generalized markup language.' Como prueba de la solidez de la norma se suele destacar el hecho de que no haya tenido que ser actualizada hasta 1994, ocho añs después de su creación. Para que se produjera el éxito»de sgml han desempeñdo un papel trascendental dos hechos:

La decisión del Departamento de Defensa norteamericano de adoptar sgml en su proyecto Cals (Computer-aided acquisition and logistic support) para la representación de información textual, con lo que se obligó a los suministradores del ejército a utilizar este formato en las descripciones técnicas de sus productos. Ha tenido como finalidad mejorar y acelerar la cadena de suministros entre el ejército y las empresas que le facilitaban productos, con especial atención a la documentación técnica. Cals desarrolló y adoptó distintos estándares, entre ellos el lenguaje sgml para la representación de información textual y los formatos gráficos Ccitt T.6 fax grupo 4 o CGM (computer graphics metafile) e Iges (initial graphics exchange specifications), diseñdos los dos ltimos para la representación de imágenes vectoriales.

Ełdesarrollo y la amplia aceptación de html (derivado de sgml) y el www en 1994. Tanto el éxito inicial de este formato como el posterior reconocimiento de sus limitaciones han hecho que un núnero creciente de editores hayan puesto su atención en sgml, que hasta entonces había sido un sistema casi desconocido por lo compleja y costosa que resultaba su utilización.

A raíz de este resultado, parece que sgml ha entrado en un ciclo positivo: existe una oferta más amplia de aplicaciones a precios asequibles, y los productores de contenidos consideran seriamente la conveniencia de migrar hacia entornos de edición y publicación basados en las directrices que establece la norma. Pero ¿qué implicaciones tiene para un productor de contenidos aceptar la norma sgml y cómo afecta a los procesos de edición? 


\section{Sgml: ciclo de producción y herramienta}

La creación de documentos sgml es costosa. En primer lugar, debe dedicarse tiempo en la fase de análisis de tipos documentales y desarrollo de DTDs. Incluso en el caso de que este paso se suprimiese adoptando un conjunto de DTDs existentes, el aprendizaje y la adaptación a un entorno de producción sgml resulta bastante trabajoso.

El proceso de intercalar etiquetas en los documentos es laborioso. La conversión automática de aquellos creados con procesadores de texto y aplicaciones de autoedición a documentos sgml utilizando las marcas de estilo del original no es suficiente y requiere la inclusión posterior de marcas para los elementos que no pueden ser inferidos a partir del formateo, si bien es verdad que algunas herramientas disponibles (Dynatag de DBT, Context-Wise de US Lynx, o EasyTag de Tetrasys) facilitan este proceso.

Siguiendo la categorización para trabajar con documentos sgml propuesta por Goldfarb [1997, p. 358], las herramientas de conversión se pueden clasificar en dos grupos:

-n-converters: conversores de documentos no sgml a documentos sgml, y

conversores entre documentos sgml o que toman a éste como fuente y lo convierten a formatos no sgml.

Los ejemplos antes citados forman parte del primer grupo.

Una vez se han creado y se les ha añdido las marcas, es necesario proceder a su validación. Este paso se ejecuta con la ayuda de un analizador o parser, que comprueba la existencia de las entidades a las que se hace referencia en el documento, si éste cumple las restricciones indicadas en su DTD y si se adecua a las reglas de producción sgml. En el caso de que el documento sea válido, se puede proceder a su composición.

Con sgml es posible incluir referencias a recursos externos utilizando declaraciones de entidades. Como recursos externos se puede indicar la propia DTD, otros archivos sgml o archivos en formatos diferentes. Generalmente las entidades se utilizan para incluir archivos gráficos o multimedia, en unión con otro componente de los documentos sgml: las notaciones.

Pero no es éste el ńico objetivo de las entidades: también se utilizan para poder trabajar con cadenas de caracteres que serán reemplazadas por otras más extensas, o para utilizar directamente los códigos ascii correspondientes a caracteres que no están disponibles en el teclado desde el cual se edita el texto.
La finalidad de la composición es generar un documento susceptible de entregarse a la imprenta o distribuirse para su lectura en línea, ocultando sus marcas y aplicándole un formateo.

Las herramientas de edición de documentos sgml incorporan utilidades para diseñr páginas de estilo. Para una misma DTD se pueden diseñr diferentes hojas de estilo. La asociación entre una DTD y sus hojas de estilo puede hacerse de distinta forma. Algunas herramientas generan un ńico archivo compilado en el que se aplican las instrucciones de formateo indicadas en dicha hoja a cada elemento del documento. Otros visores son capaces de generar la presentación del documento sgml con el formato que establezca la hoja de estilo asociada de forma dinámica, sin un proceso de compilación previo.

Se han desarrollado normas que guían en la creación de hojas de estilo para documentos sgml: Os (output format), desarrollada en el marco del proyecto Cals, o la norma internacional ISO 10179 Dsssl (document style semantics and specification language). El propósito de Dsssl es más ambicioso que el de $O s$, ya que no sólo pretende resolver el tema del formateo de los textos sgml, sino que también especifica los procesos de conversión de documentos de una DTD a otra.

En la fase de distribución del documento es donde se pueden obtener las máximas ventajas de trabajar con sgml. Frente a las alternativas basadas en marcas descriptivas, los documentos sgml pueden llegar a ser procesados por la aplicación que los recibe, lee o interpreta. Por ejemplo, los distintos elementos se podrían utilizar para alimentar una base de datos relacional, bibliográfica, etc.

De cualquier forma, si bien sgml ofrece grandes ventajas, la dificultad que implica el proceso de creación de estos documentos y la alta curva de aprendizaje a la que están asociados constituyen los principales escollos que deben afrontar las organizaciones para adoptar este formato.

La opción semántica no sólo está presente en sgml, sino que también constituye buena parte de la base de otros sistemas, como ODA/Odif (open document architecture/open document interchange format). $\mathrm{Y}$ es la filosofía subyacente en las transacciones EDI (electronic data interchange) que utilizan mensajes (tampoco en este caso se trata de un ejemplo típico de edición digital) ajustados normalmente a las normas Edifact (ISO 7372 y 9735) ó Ansi X.12.

\section{Sistemas DIP}

Paralelamente a los modelos que hacen uso de la opción semántica, se fue desarrollando una serie de programas que se englobaron bajo el nombre DIP (do- 
cument imaging processing). Se trata de softwares que han jugado un papel importante en una fase de la ofimática, pues sirven para la gestión de archivos digitales con las imágenes escaneadas de los documentos manejados por las diversas organizaciones.

A lo largo de la historia, las empresas involucradas en este ámbito (FileNet, Olivetti, Xerox, Wang, etc.) han hecho uso de diferentes formatos gráficos, algunos ajustados a normas; otros desafortunadamente propietarios, lo cual les hizo incompatibles, con los consiguientes problemas para ulteriores migraciones de software. Segú el formato utilizado, se hace mayor o menor uso de sistemas de compresión. Tiff (tagged image file format) es el que ha alcanzado una mayor popularidad en la representación de los documentos mediante imágenes de tipo raster, aunque podríamos incluir otros como jpeg (join photograph experts group), gif (graphics interchange format), etc.

Como es natural, los documentos, además de texto, pueden contener tablas, gráficos vectoriales e imágenes, pero con la peculiaridad de que todos estos componentes constituyen un ńico archivo y no pueden tratarse separadamente. Esto presenta serias dificultades: cada página escaneada conforma un archivo independiente, por lo que si el documento consta de varias resulta necesario agruparlas de alguna forma (guardándolas en un mismo directorio o ensamblándolas utilizando alguna aplicación que ofrezca esta funcionalidad). La imagen escaneada debía ser procesada por un OCR (optical character recognition) para poder tratar sobre su contenido y había que asignar una serie de propiedades identificativas y descriptivas que se almacenaban en una base de datos externa al documento.

\section{Documentos compuestos estáticos}

Actualmente la representación del documento como imagen puede considerarse un precedente histórico de los formatos de réplica o portables: Acrobat$P D F$, Envoy, DigitalPaper, etc. Sus proveedores han dado un gran paso adelante con la comercialización de productos que permiten obtener una representación en su formato propietario que va más allá del mero resultado de escanear un documento impreso.

La necesidad de este tipo de formatos hizo surgir el estándar ODA/Odif recogido en la norma ISO 8613 . Su historia es la historia de un fracaso: comenzó a elaborarse en 1982, y entonces las O'iniciales correspondían a Office (más tarde Open). Su objetivo era conseguir que lós documentos, tanto textuales como gráficos, pudieran ser transferidos, con todos sus atributos intactos, de un sistema a otro para poder ser luego editados, procesados, almacenados, impresos y transmitidos."Se pretendía, en definitiva, solucionar el proble- ma de comunicación entre formatos de procesadores de texto propietarios, cuya proliferación se veía en aquellos momentos (más que ahora) como una dificultad para el intercambio de documentos complejos. Usando ODA/Odif como formato de comunicación intermedio, el sistema que recibiera un texto convertido desde otro software podría presentarlo en pantalla y gestionarlo como si él lo hubiera creado.

La norma recibió un fuerte impulso en 1991, fecha en la que se creó el $O D A$ Consortium, constituido por Bull, DEC, IBM, ICL, Siemens Nixdorf, agregándose después otros como WordPerfect. El consorcio desarrolló un conjunto de herramientas software, que se distribuyeron gratuitamente, para transformar documentos al formato de intercambio Odif, para que a continuación se pudieran transferir de nuevo esos documentos a cualquier otro sistema que diera soporte a $O D A$.

A diferencia de sgml dende el énfasis se centra en la representación del contenido y la estructura del documento- $O D A$ codifica, además de la estructura lógica del documento, el formateo que se debe aplicar para su impresión y visualización en pantalla. La norma define un modelo de documento constituido por tres estructuras: la lógica que podría ser equiparable a la $D T D$ de sgml, ta física grupos de páginas, páginas, marcos y bloques, $y$ el contenido propiamente dicho, el cual constaría de texto (codificado de acuerdo con los sistemas ISO 8859 e ISO 6937), imágenes tipo bitmap (raster) y gráficos vectoriales. Para la representación de gráficos se adoptó la utilización de los formatos CGM (normalizados en ISO y Ccitt fax grupos 3 y 4 ).

Esta complejidad obligó a desarrollar distintos perfiles de la norma, denominados document application profiles (DAP), entre ellos el Fod11, Fod26, Fod36, Fod112, etc. Los podríamos definir como niveles de conformidad con la norma estandarizados y compatibles entre sí. A pesar de que su Ítima revisión se hizo hace sólo cuatro añs, el formato ha caído en el olvido más absoluto. La actividad del ODA Consortium cesó, y el soporte y desarrollo de productos acordes con la norma también se vieron paralizados. Quizá su complejidad fue una de las causas por las que ODA/Odif no consiguió alcanzar un nivel de aceptación suficiente entre los fabricantes de software. También es verdad que el predominio logrado por Microsoft y sus aplicaciones de sobremesa en los lítimos añs fueron los principales enemigos para su éxito. Nos encontramos ante un ejemplo que demuestra que, en el ámbito de la normalización, no siempre alcanzan el éxito las mejores normas y que en su adopción juegan un papel importante otros factores como la posición en el mercado de sus impulsores. 
Ante tal fracaso, las empresas especializadas en software para la gestión de documentos digitales se enzarzaron en una carrera para ver quién conseguía imponer como estándar su propio formato de intercambio. Así surgió en 1993 Acrobat, un sistema con el que Adobe ha facilitado la difusión de documentos digitales entre sistemas software y hardware que son incompatibles entre sí. Haciendo uso de la tecnología Acrobat es posible ver en la pantalla del ordenador o imprimir (con gran fidelidad a la tipografía, estilo, gráficos y colores del original) documentos que pueden haber sido creados en un sistema completamente incompatible.

\section{«Adobe era la única cuyo soft- ware de edición electrónica ha ocupado siempre un lugar des- tacado en su línea de produc- tos, situándose el resto en un puesto secundario dentro de sus ofertas comerciales»}

Además del formato propio de Acrobat (PDF) hay que citar DigitalPaper de Common Ground (antes NoHands Software), Envoy de Tumbleweed, Replica de Farallon, etc., que ya han sido abandonados. Estos formatos, denominados también de réplica, son legibles en distintas plataformas hardware y sistemas operativos, siempre que se disponga de un lector especial distribuido (generalmente de forma gratuita) por su fabricante. Normalmente contienen dentro de un solo fichero textos, tablas, gráficos, etc., haciendo uso de sistemas de compresión. Permiten la presentación formal definitiva (en pantalla o impresión) y el intercambio (portabilidad) de los materiales para su visualización.

Los documentos de réplica se crean mediante un proceso de conversión, utilizando un software especial que actú como un controlador (driver) de impresión. Para generar un documento basta con imprimirlo desde la aplicación con la que se creó (Microsoft Word, WordPerfect, QuarkXpress, FrameMaker, Excel, etc.) tras haber seleccionado el controlador correspondiente desde el cuadro de diálogo que contiene las opciones de impresión.

El documento resultante mantiene las características tipográficas y el formateo del original, y todos los objetos que lo componen se fusionan en un ńico archivo comprimido, con lo que se minimiza el espacio de almacenamiento requerido y se facilita su transmisión a través de redes. El formato también almacena información sobre las fuentes utilizadas en el documento original. En el caso de que el ordenador desde el que se lee no disponga del tipo de letra utilizado al crearlo, el software lector es capaz de emularla de forma razonablemente satisfactoria. Tras la conversión es posible añdir al documento tablas de contenidos, crear índices en texto completo, hiperenlaces, etc., así como indicar restricciones de seguridad. Esta edición se lleva a cabo utilizando aplicaciones proporcionadas por el fabricante del formato.

Para leer y editar los documentos es necesario disponer de un software propietario. ḱta es una de las características más importantes de los formatos de réplica: sólo pueden leerse o imprimirse si se dispone de un programa especial. Algunos sistemas, como Envoy Run-Time, Replica Viewer, o MiniViewer de Common Ground, fusionaban los documentos con el visor en un ńico archivo ejecutable, con lo que no es necesario poseer el visor para poder leerlo.

Las posibilidades de edición disponibles con este tipo de formatos son muy limitadas (eliminación e inserción de páginas extraídas de otro archivo en el mismo formato, creación de notas e hiperenlaces, etc.) y no suelen estar disponibles en los lectores de libre distribución.

En la lista de formatos compuestos estáticos se podría incluir también RealPage, desarrollado y utilizado por la editorial inglesa Catchword que, desde 1994, se dedica en exclusiva a la edición digital de publicaciones académicas. La orientación de negocio de la empresa difiere de la del resto de productores de formatos de réplica: no pretende vender software, sino servicios de hosting (alojamiento) para editoriales que faciliten la distribución electrónica de documentos, gestionando el proceso de conversión a partir de los documentos suministrados por los editores y su distribución a través de servidores dedicados, así como el control y la facturación de los accesos a las publicaciones. Real Page requiere un visor especial, disponible ńicamente para Windows y a través de un applet Java para cualquier navegador internet.

\section{EI impacto de internet en los formatos de réplica}

La generalización de la Red y de las intranets ha obligado a los fabricantes de los formatos de réplica a estudiar las posibilidades de integrarse con el lenguaje html. La adaptación al nuevo entorno se ha centrado en las siguientes líneas de trabajo:

Optimización de los formatos para lograr representaciones de los documentos que ocupen menor espacio, para así facilitar su distribución a través de la Red.

Pesibilidad de crear enlaces que tengan como destino documentos html. 
Besarrollo de plug-ins y controles ActiveX que permitan leer documentos en formato propietario desde cualquier navegador internet.

Đistribución de documentos a través de servidores web página a página: enviándolas una tras otra a medida que las solicita el software cliente, sin que sea necesario descargarlas todas para poder comenzar a leerlo. Esta posibilidad se lleva a cabo mediante scripts CGI ejecutados por el servidor web a través del cual se accede a los documentos.

Este sistema de distribución fue adoptado inicialmente por Adobe en su especificación Amber, que constituyó la base para la versión 3.0 liberada el añ 1996. Posteriormente lo adoptaron otros fabricantes.

\section{Adobe y sus competidores}

En el apartado anterior hemos citado formatos portables desarrollados por distintas compañas. Hasta hace poco tiempo podía considerarse a algunos de ellos alternativas al que había logrado una mayor popularidad: PDF, que siempre ha ocupado una posición predominante. De las empresas citadas, Adobe era la ńica cuyo software de edición digital ha ocupado siempre un lugar destacado en su línea de productos, situándose el resto en un puesto secundario dentro de sus ofertas comerciales. Por ejemplo, la línea de trabajo de Farallon y Hummingbird, recientemente adquirida por $P C$ Docs se centró en el desarrollo de software para la conectividad entre sistemas hardware heterogéneos.

\section{«Con sgml se pueden crear hi- perenlaces que permiten reco- rrer el documento de forma no lineal. Pero hay una limitación: sólo es posible crear hiperenla- ces que unan dos partes del mismo archivo»}

En 1998 Adobe Acrobat y PDF dejaron de tener competencia: Farallon abandonó la tarea de desarrollar y comercializar Replica; Hummingbird había hecho pb́lica su decisión de hacer lo mismo con Common Ground en febrero de ese mismo añ y Tumbleweed (encargada de desarrollar Envoy) orientó su actividad hacia la distribución segura de documentos, recomendando el uso de PDF, al que definió como estándar de facto en una nota de prensa publicada en octubre de 1997.

Esto no debe interpretarse como una superioridad técnica del formato PDF frente al resto de sus competidores. Simplemente se trata, nuevamente, de una mayor aceptación debida sobre todo a la posición de superioridad que Adobe tenía en el mercado, gracias en buena parte a la importante implantación del estándar de facto PostScript, que había sido lanzado por la compañ en 1985.

\section{Formatos para el intercambio}

La necesidad de intercambiar documentos entre aplicaciones diferentes, y en la mayoría de los casos incompatibles, ha sido uno de los principales problemas que ha tenido que afrontar la aplicación de las tecnologías informáticas dentro de la gestión documental.

Para resolverlo se han diseñdo distintos formatos. Algunos de ellos han sido propuestos por los fabricantes de procesadores de texto o herramientas de autoedición, como por ejemplo RTF (rich text format) de Microsoft, MIF (maker interchange format) de Adobe o CDA (compound document architecture) de Digital. A excepción de este ĺtimo, que proponía un modelo más complejo y sofisticado, en el resto de casos se trata de formatos que descartan la utilización de las características de los originales que los hacen incompatibles, a favor de un modelo de representación más simple para el que se pueden diseãr filtros de conversión con mayor facilidad.

Estos formatos están pensados para ser reconocidos por aplicaciones distintas a la aplicación con la que fueron creados (siempre que incorporen un filtro de conversión entre ellos), ser convertidos al formato propietario para poder ser editados y de nuevo poder ser almacenados en el formato de intercambio.

Con los formatos orientados al intercambio:

es-posible siempre la edición de un documento,

ła-conversión es bidireccional (contrariamente a lo que sucede con los formatos portables, en los que sólo hay conversión en una dirección), y

no es necesario disponer de una aplicación especial para leer los documentos, ya que su objetivo es que el resto de aplicaciones sean capaces de interpretar el formato.

\section{EI paradigma hipertexto y el www}

Los sistemas hipertexto son una de las asignaturas pendientes de la edición digital. El término paradigma y conceptos como transclusión, lectura no secuencial, etc., han contribuido a crear unas expectativas mayores a lo que podríamos considerar tan sólo como una inestimable ayuda para la lectura de grandes textos: la posibilidad de unir sus partes mediante enlaces. De hecho, las implementaciones reales de sistemas hipertexto distan mucho de las concepciones visionarias auguradas por sus precursores en la década de los cincuenta y sesenta: Vannevar Bush, Ted Nelson, etc. 
Bajo la denominación hipertexto encontramos distintas aplicaciones bastante dispares y basadas en formatos o lenguajes de marcas propietarios capaces de ser interpretados por lectores especiales: HyperCard, Folio, etc. Con sgml se pueden crear hiperenlaces que permiten recorrer el documento de forma no lineal. Pero hay una limitación: sólo es posible establecer hiperenlaces que unan dos partes del mismo archivo. Para crear un vínculo entre dos zonas de un documento sgml es necesario definir su origen y su destino. El origen se marcará utilizando un elemento especial, al que se llamará por convención link o xref, que contará con un atributo ref de tipo idref. Este atributo tomará como valor el nombre que se haya dado al punto destino del enlace, el cual se declara como un atributo con nombre target de tipo $i d$.

Un parser identificará los orígenes del hiperenlace y comprobará si existen las declaraciones de los destinos a los que apuntan. Si se necesita crear uno que una dos archivos, es preciso recurrir a recursos ofrecidos por la norma HyTime, aprobada como estándar en 1986.

Pero desde el ã̃ 1992 hablar de hipertexto equivale casi a hablar del web, del lenguaje html y sus derivados. $\mathrm{Html}$ es una aplicación del lenguaje sgml que indica cómo se deben codificar documentos para distribuirlos en el www. Independiente de plataformas hardware o software, html parecía ser la solución idónea a los problemas de intercambio de documentación digital. Pero pronto se hicieron manifiestas sus lagunas:

incapacidad para mantener las características tipográficas y el formateo de los documentos,

elevados costes en ancho de banda y dificultades en la transmisión de documentos compuestos,

earencia de fórmulas de compresión asociadas al formato,

inexistencia de mecanismos de acceso a la información (tablas de contenidos, índice de palabras clave, etc.).

Por otra parte, la evolución de html en los lítimos añs ha estado condicionada por la presión ejercida por Netscape y Microsoft, fabricantes de los navegadores internet más utilizados. El W3C, encargado de normalizar y controlar la evolución de los estándares para el www, se vio desbordado por los desarrollos de estas dos empresas, que añdían nuevas etiquetas al formato aprobado como estándar y diseãban módulos y lenguajes de script que sólo podían ser interpretados por sus respectivos navegadores.

Para resolver estas limitaciones se han diseñdo distintas alternativas: el lenguaje html dinámico (dhtml), el desarrollo html-help (propietario de Micro- soft y propuesto al W3C para su inclusión en el estándar) y el lenguaje xml. Esta Ítima opción se perfila como la alternativa más fuerte en el panorama de la edición digital y acapara la mayor atención por parte de fabricantes de software y usuarios.

\section{«Frente al conjunto de etiquetas predefinidas que conforman html, xml ofrece la posibilidad de definir nuevas marcas para codificar la estructura y conte- nido de los distintos tipos de documentos»}

1. Dhtml. El término dhtml se utiliza para hacer referencia a una serie de características soportadas por la versión 4 del Internet Explorer y Netscape Communicator. Algunas de estas particularidades se tomaron de los borradores de la que iba a ser la nueva versión de html, la 4.0, cuyo desarrollo se llamó Proyecto Cougar.

El objetivo final de $d h t m l$ se centra en lograr una mayor interacción en las páginas html, minimizando los intercambios de datos entre el cliente y el servidor web, y permite:

un mayor control sobre los elementos que conforman una página html, mediante un modelo jerárquico de descripción de documentos (document object model) y lenguajes de scripts,

pesibilidad de aplicar formato al contenido de una página y, lo que es más importante, modificar el formato desde el navegador en respuesta a acciones ejecutadas por el usuario,

eentrol de la posición de los objetos en una página y posibilidad de desplazarlos por ella, y

eapacidad para cambiar su contenido una vez ha sido descargada del servidor.

Para lograr estas mejoras se han reunido distintos desarrollos: las hojas de estilo (cascading style sheets) que especifican cómo se deben formatear y posicionar los elementos en una página y lenguajes de script capaces de interpretar la jerarquía de objetos DOM.

Sin embargo, la normalización no se ha alcanzado. Netscape Navigator e Internet Explorer no utilizan modelos idénticos para las hojas de estilo y siguen existiendo distintos lenguajes de script: Vbscript y $J_{S}$ cript (de Microsoft) y Javascript (de Netscape), aunque en este punto se ha alcanzado cierta homogeneidad a partir de la decisión de la Ecma (European Computer Manufacturers Association) de adoptar este Itimo como estándar, y la apuesta de Microsoft a favor de su versión de Javascript en detrimento de VBscript. 
A pesar de que se trata de un gran avance, dhtml es un formato excesivamente orientado hacia la presentación de páginas y a los efectos visuales, con serias dificultades para representar el contenido semántico y la lógica de la información.

\section{Html compilado: html-help}

El excesivo tamañ de los documentos html y de sus componentes (gráficos, animaciones, etc.) así como la necesidad de aplicar mecanismos de acceso, ha llevado a Microsoft a proponer un formato propietario: $h t m l-h e l p$, que consiste en páginas html compiladas en un ńico archivo. Su lanzamiento se anunció en la WinHelp Conference de 1996, y la primera versión de prueba (llamada beta 1) se distribuyó a través del web site de Microsoft el 27 de noviembre de 1996.

El formato permite realizar bśquedas en texto completo y ofrece las opciones de navegación que caracterizaron el sistema de edición hipertexto WinHelp, desarrollado por Microsoft para distribuir ayuda en línea para los programas que se ejecutasen en el sistema operativo Windows (tablas de contenidos, palabras clave, etc). Html-help está concebido como el nuevo formato para la distribución de documentación en línea para aplicaciones software, sustituyendo a WinHelp e incluso al que había sido el sistema utilizado por $M i$ crosoft para la edición digital de grandes volmenes de documentación: Media View.

Pero html-help presenta una limitación: sólo puede ser interpretado por equipos que dispongan del navegador Internet Explorer. A pesar de que puede considerarse como una alternativa válida para la edición digital y fue propuesto al $W 3 C$ para su inclusión en el estándar html, su utilización parece quedar restringida a la publicación de documentación técnica de software.

\section{Xml: el futuro de la edición digital}

En menos de tres añs ha pasado de ser una alternativa a html a convertirse en el formato con mayores perspectivas de acaparar el mercado de la edición digital y el desarrollo de aplicaciones internet/intranet. Su evolución comenzó en septiembre de 1996, auspiciado por el W3C con un claro propósito: diseñr un lenguaje de marcas optimizado para ser utilizado en internet, que combinase la simplicidad de html con la capacidad expresiva de sgml.

En su definición participaron Microsoft, IBM, Sun Microsystems, Novell y Hewlett Packard. La versión 1.0 fue ratificada por el $W 3 C$ en la conferencia sobre sgml/xml celebrada en Washington en diciembre de 1997.

Las principales características de este formato son: extensibilidad, formateo, gestión avanzada de los hiperenlaces y modularidad.

1. Extensibilidad. Xml es un perfil de sgml y no una aplicación, como es el caso de html. Frente al conjunto de etiquetas predefinidas que conforman html, $\mathrm{xml}$ ofrece la posibilidad de definir nuevas marcas para codificar la estructura y contenido de los distintos tipos de documentos. Al igual que sucede con sgml, es posible crear DTDs que indiquen las reglas que debe cumplir cada tipo de documento y que faciliten su tratamiento automatizado por cualquier aplicación capaz de interpretar la $D T D$.

La viabilidad de marcar los elementos informativos permite procesar el documento en el puesto cliente. Xml, unido al lenguaje Java, hace realidad la utilización de los navegadores como clientes universales válidos para cualquier tipo de aplicación.

2. Formateo. Ofrece un mecanismo que permite indicar cómo se deben formatear, imprimir y mostrar en pantalla los distintos elementos de un documento. Estas hojas de estilo se diseñrían de acuerdo con las especificaciones $\mathrm{Xsl}$ (equivalente a las cascading style sheets del lenguaje html).

3. Gestió de hiperenlaces . Xlink es el modelo propuesto en xml para la gestión de hiperenlaces. El tratamiento es similar al utilizado por sgml, aunque también se incorpora la utilización de URLs característica de html. Las principales mejoras frente al modelo usado en html son: la posibilidad de trabajar con enlaces bidireccionales, con vínculos que, partiendo de un mismo origen, alcancen dos o más puntos destinos y la transclusión del destino del enlace en el documento origen.

4. Modularidad. Un documento xml puede ser modular gracias a la noción de entidades heredada de sgml y podrá estar formado por distintos archivos xml que se presentarán en pantalla como si se tratase de un ńico documento.

\section{Xml y sgml}

Muchas de estas ventajas, quizás con la excepción del tratamiento de los hiperenlaces, ya se encontraban en sgml. ¿Por qué xml y no sgml?

El primero es una versión simplificada del segundo, del que se han eliminado aquellas características poco utilizadas que añdían una complejidad en ocasiones insalvable para los fabricantes de software que afrontan el diseñ de software para la edición con sgml. 


\section{Content}

\section{Access}

\section{Utility}

\section{IDE/}

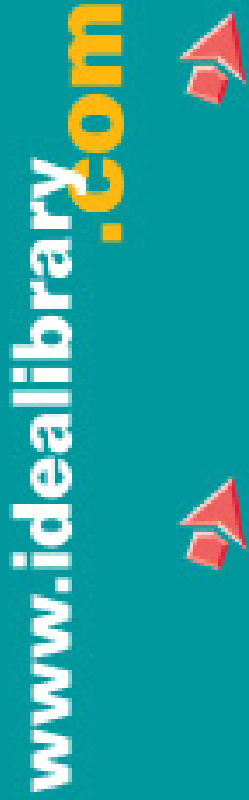

Joumals now on IDEAL number nearly 250 titles from Academic Press and Harcourt Health Sciences - including Churchill Livingstone, W.B. Saunders, Ltd. and Baillière Tindall. In 2001, more titles from W.B. Saunders and titles from Mosby will be introduced. Joumal backfiles now on IDEAL include AP titles from 1993 forward and selected HHS medical titles beginning with 1999 issues.

IDEALReferenceWorks, an online collection of encyclopedias, continues to grow on IDEAL. For 48-hour free guest access to any IDEALReferenceWorks title and further information on the collection, visit: www.idealibrary.com/idealreferenceworks

IDEALReferenceWorks now offers or will soon offer:

- Encyclopedia of Biodiversity

- Encyclopedia of Food Microbiology

- Encyclopedia of Forensic Sciences

- Encyclopedia of Human Nutrition
- Encyclopedia of Immunology, Second Edition

- Encyclopedia of Separation Science

- Encyclopedia of Spectroscopy \& Spectrometry

- Encyclopedia of Virology, Second Edition

SciVision, presenting leading-edge electronic information systems, is now also available to IDEAL users. SciVision produces high-quality scientific-application sofitware tied to smart databases and comprehensive toolsets, to help research and development professionals, educators and students focusing on the biomedical, environmental and materials sciences. For more information, visit www.scivision.com

SciVision now offers these information systems:
- BiomedSource ${ }^{\mathrm{TM}}$
- SciGLASSB
- oncols ${ }^{\mathrm{ru}}$
- toxSYSim
- qsarlSTm

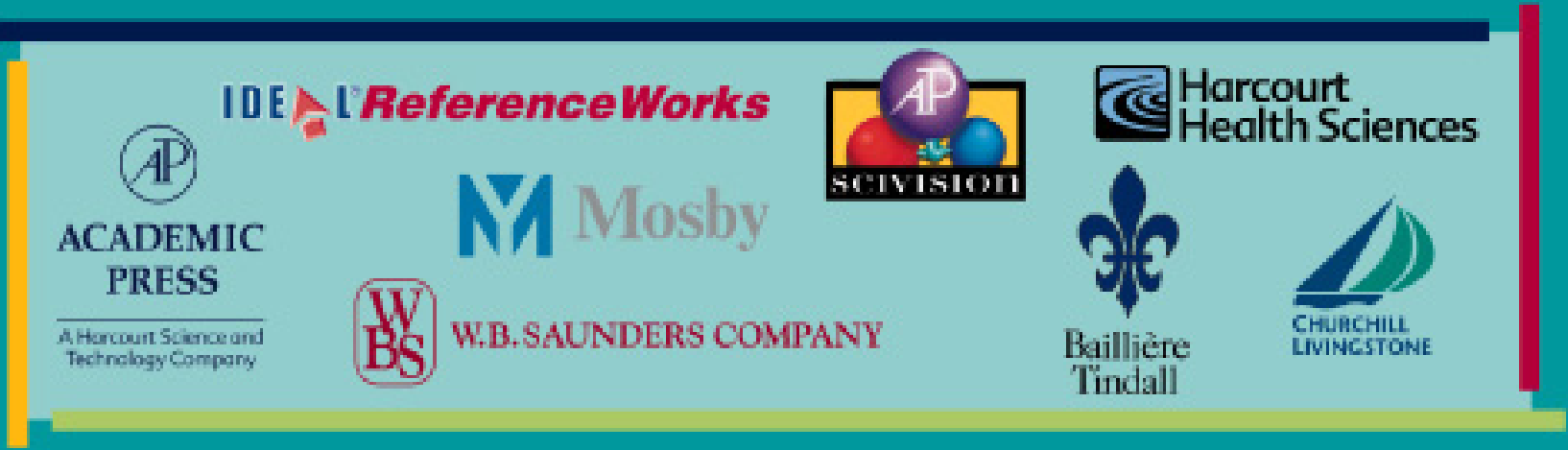

IDE/CLAleVt

This popular, free tables-of-contents e-mailing service remains awailable at: www.academicpress.com/ideal-alert 
La distribución de documentos se simplifica al ofrecer la opción de distribuir o no la DTD que utiliza un documento junto a éste. El motivo es justificado: segú el tratamiento que se quiera hacer en el ordenador receptor del documento, la transmisión de la $D T D$ y los procesos de validación pueden suponer mayores tiempos de transferencia y costes de ancho de banda innecesarios. Conviene seãlar que xml es compatible tanto con sgml como con html y en un futuro las aplicaciones internet harán un uso conjunto de ambos.

\section{«En un entorno docucéntrico los formatos y la estructura de alma- cenamiento interno de los com- ponentes de un programa son transparentes para el usuario»}

Actualmente los fabricantes de aplicaciones para la edición digital ya soportan xml: DBT, Dataware, NextPage, ArborText, Interleaf, etc. Microsoft incorpora $\mathrm{xml}$ a su navegador y ofrece un parser escrito en Java (Msxml) para comprobar la validez de los documentos. Ya contamos con gran cantidad de aplicaciones que hacen un uso intensivo del nuevo formato: por ejemplo, $R D F$ (resource description format) para la descripción de documentos disponibles en el web; $C D F$ (channel description format), desarrollado por Microsoft para transmitir información a través de los canales push del Internet Explorer; MathML (mathematical markup language); OFX (open financial exchange) para el intercambio de datos financieros; OSF (open software description), creado por Microsoft y Marimba para describir paquetes de instalación de aplicaciones software...

\section{Tecnologías para la interacción}

En los párrafos anteriores hemos hecho un resumen de los principales tipos de formatos disponibles para la edición digital. A continuación dedicamos dos apartados a las tecnologías utilizadas para la interacción y compatibilidad de documentos digitales: OLE (object linking and embbeding) y Opendoc, para la creación y edición de documentos dinámicos; y los visores universales, capaces de mostrar documentos en distintos formatos sin necesidad de hacer ninguna conversión previa.

1. Documentos compuestos dinánicos. Están formados no sólo por texto, sino también por gráficos, tablas, componentes multimedia, etc., creados con distintas aplicaciones y ensamblados posteriormente. Los componentes que lo conforman se almacenan en archivos independientes que pueden ser edi- tados con distintas utilidades y reutilizados en un nú mero ilimitado de documentos. Los cambios que se hagan en cada componente se reflejarán en el documento del que forman parte. De ahí la denominación dinámico.

Conviene aclarar que no es un formato especial para guardar y distribuir documentos, sino unas tecnologías que permiten ensamblar componentes en distintos formatos (textual, gráfico, etc.) y el usuario podrá editarlos sin necesidad de abrir la aplicación capaz de interpretar su formato.

Estas tecnologías están vinculadas a las interfaces gráficas de usuario y fueron el desencadenante de lo que se llamó sistemas operativos docucéntricos. Este término se utiliza para indicar que el documento, y no las aplicaciones, es el protagonista de la informática personal y de la ofimática, como confirma la metáfora del escritorio utilizada por Macintosh, por Windows y por las interfaces de usuario características de los sistemas de gestión documental (Documentum, Docs Open, FileNet, etc.). En un entorno docucéntrico los formatos y la estructura de almacenamiento interno de los componentes de un programa son transparentes para el usuario.

Las dos tecnologías más importantes para la integración de componentes en documentos compuestos dinámicos son $O L E$, de Microsoft, y Opendoc, desarrollada inicialmente por un consorcio del que formaron parte IBM, Apple, Lotus, Adobe y Novell-WordPerfect.

Este grupo de empresas creó los CILabs (Component Integration Laboratories), encargados de desarrollar las especificaciones del modelo. Entre éstas se encuentra el subsistema de almacenamiento (Bento de Apple), el de intercambios de mensaje entre objetos (System Object Model de IBM), lenguajes de scripts e incluso una interface que permite incrustar objetos OLE en documentos Opendoc (componentGlue), etc.

De nuevo la preponderancia de Microsoft ha marcado sus destinos: los creadores del estándar Opendoc

\section{Versión online de EPI}

Existe una versión electrónica de la revista El profesional de la información, de uso gratuito para la mayoría de los suscriptores (empresas, organismos, instituciones), consultable en:

http://www.swetsnet.nl/

Más información en:

http://www.swets.nl/sps/journals/jonline.html 
se vieron obligados a dar soporte a la tecnología propietaria de la competencia, para pasar a continuación a plegarse ante el éxito de MS OLE y abandonar el desarrollo de Opendoc.

$O L E$ es la base del modelo de documento compuesto dinámico utilizado por Microsoft. Su origen se remonta a 1991 y surgió por la necesidad de incluir gráficos creados con Microsoft Graph en presentaciones PowerPoint. OLE ofrece dos mecanismos de integración de componentes en un documento compuesto: embbeding y linking. En el primer caso el componente sólo puede ser accedido desde el documento contenedor, mientras que en el segundo tiene existencia independiente. La tecnología $O L E$ ha alcanzado un gran impacto y ha evolucionado hasta convertirse en la infraestructura tecnológica por excelencia para la informática basada en componentes y la orientación a objetos.

2. Visores universales. Los fabricantes de documentos digitales han desarrollado visores que permiten leer e imprimir los documentos creados con sus aplicaciones, por ejemplo FrameViewer de Adobe para FrameMaker o WorldView de Interleaf. Estos visores, aunque sólo permiten trabajar en modo lectura con un ńico formato, pueden considerarse una alternativa a los lenguajes de réplica si se trata de distribuir documentos digitales que hayan sido creados con una aplicación propietaria.

Incorporan utilidades para la compresión y encriptación. Frente a estos visores asociados a un ńico formato, los universales no requieren la conversión previa a uno propietario (interpretan directamente los archivos fuente), mantienen las características tipográficas del original y se han convertido en un componente clave en los sistemas de gestión documental y en un complemento de gran utilidad para las aplicaciones de mensajería digital y los navegadores internet. En esta línea de productos se encuentra KeyView Pro, desarrollado por FTP Software y actualmente propiedad de Verity, o Quick View Plus de DBT, o Autovue.

\section{Conclusiones}

En los apartados anteriores hemos ofrecido una breve descripción de las iniciativas más importantes que han contribuido a la homogeneización de los formatos para la edición digital y a lograr mayores posibilidades de interacción entre aplicaciones. Uno de los principales objetivos del texto es presentar las tendencias observadas como alternativas complementarias.

A lo largo del ciclo de vida de un documento, y dependiendo del uso que se le quiera dar en cada fase, se pueden utilizar distintos formatos para aprovechar las ventajas que ofrece cada uno de ellos. Así, los sistemas para la gestión de documentos digitales aplican el formato original del documento para su edición, formatos de réplica en las fases de validación, o html para su distribución a través del web.

Algunos analistas auguran un futuro en el que sgml será sustituido por su versión simplificada, $\mathrm{xml}$, al que califican como un formato integrador en el ciclo de vida de los documentos: desde su producción hacia su distribución. Otros se resisten a aceptar xml como una alternativa real a sgml.

Cuestionar la importancia de xml y su creciente protagonismo en los próximos añs resultaría ingenuo. Sin embargo, la producción de documentos estructurados implica elevados costes, y xml ha de afrontar, en su camino hacia la supremacía, las mismas dificultades que han frenado el desarrollo de sgml a lo largo de los ĺtimos añs, si bien en un mercado ya mucho más dinámico. La sustitución de html por xml también parece algo lejana.

El protagonismo de xml hay que buscarlo en aplicaciones específicas que utilicen internet como red de transmisión y que requieran: tratamientos complejos de los documentos e integración de datos entre aplicaciones diferentes. El comercio electrónico es el ejemplo más significativo del tipo de aplicaciones que van a sacar partido de este formato.

En el suministro de documentos, xml va a tener dificultades para demostrar sus ventajas frente a la facilidad de creación y conversión asociada a los formatos portables, en gran parte debido a la eliminación de barreras entre editores, distribuidores y usuarios que limita las necesidades de procesamiento en local. No obstante, algunos proveedores de contenidos ya han empezado a utilizarlo en la difusión push de información para integrar fuentes internas con información externa (por ejemplo, Dow Jones con su Intranet Toolkit).

$\mathrm{Xml}$, al igual que sgml, ofrece la ventaja de poder compartir información en formato estructurado para facilitar su procesamiento en distintos centros de tratamiento de datos o con aplicaciones heterogéneas. Si esta necesidad no existiera, trabajar con xml puede acabar convirtiéndose en algo tan costoso como sgml.

\section{Pedro Hípola \\ phipola@ugr.es}

Ricardo Eíto Brun

ricardo.eito@adecco.es 